\title{
Broadcasting communication in high degree modified chordal rings networks
}

\begin{abstract}
The design of the interconnection network is one of the main research issues in distributed computing with regard to some specific parameters. This paper works on Modified Chordal Rings Degree Six (CHRm6) topology. Two broadcasting schemes for CHRm6 are proposed. The first scheme is for even source nodes and the second scheme is for odd source nodes. The proposed broadcasting schemes give upper and lower bound of broadcasting in CHRm6 depends on total number of nodes. We prove the lower bound on the broadcast time is $\mathrm{d}+2$ where $\mathrm{d}$ is the diameter of the CHRm6.
\end{abstract}

Keyword: Chordal rings; Interconnection; Broadcasting; Topology; Delay; Broadcast time 